\title{
Transplantation of congenitally opaque corneas
}

\author{
Beatrice E Frueh, Stuart I Brown
}

\begin{abstract}
Aims-To assess retrospectively the prognosis and complications of corneal grafting for congenital opacities.

Methods-Fifty eight eyes of infants and young children with congenital corneal opacities were studied retrospectively. Preoperative diagnoses included sclerocornea (27 eyes), Peters' anomaly (17 eyes), partial sclerocornea (12 eyes), and congenital glaucoma (two eyes). Penetrating keratoplasty was performed between 5 days and 65 months of age with a mean follow up of 40 (SD 29) months.

Results-The overall success (including regrafts) was $70 \%$ in eyes with sclerocornea, $83 \%$ for partial sclerocornea, and $100 \%$ for Peters' anomaly. However, 23 eyes had to be regrafted between 2 weeks and 110 months postoperatively. The probability of maintaining a clear graft, calculated by survival analysis, was $75 \%$ (SE 6\%) at 1 year and $58 \%(7 \%)$ at 2 years for the entire group. Complications included cataract development (12 eyes), secondary glaucoma (14 eyes), epithelial defects (six eyes), band keratopathy (five eyes), retinal detachment (three eyes), wound leakage (two eyes), retrocorneal membrane (one eye), and microbial keratitis (two eyes).

Conclusions-It is concluded that corneal grafting for congenital opacities in infants has an excellent potential for long term survival and should be performed as early as possible for unilateral as well as bilateral involvement. The postoperative course is complex and often requires regrafting.

(BrF Ophthalmol 1997;81:1064-1069)
\end{abstract}

Department of

Ophthalmology,

University of

California, San Diego,

La Jolla, California,

USA

B E Frueh*

S I Brown

${ }^{\star}$ Current address:

Department of

Ophthalmology, University

of Bern, 3010 Bern,

Switzerland.

Correspondence to:

Stuart I Brown, MD, UCSD,

Shiley Eye Center, 9500

Gilman Drive La Jolla, CA

92093-0946, USA.

Accepted for publication 27 May 1997
Over the past two decades, a better understanding of wound healing of infant corneal transplants and modifications of surgical techniques have contributed to a substantial improvement in the prognosis of paediatric penetrating keratoplasties (PKP) for congenital corneal opacities. ${ }^{1-3}$ The first anecdotal reports of successfully transplanted corneas in Peters' anomaly and sclerocornea appeared in the 1970s. ${ }^{4-7}$ Since then, larger series with promising results have been published. Schanzlin et al reported clear corneal grafts from 3 to 30 months postoperatively in nine of 15 eyes with various types of congenital corneal opacities. ${ }^{8}$ This study emphasised the use of large donor corneas and early suture removal. Stulting et $a l^{2}$ calculated the probability of maintaining transparency at 1 year after corneal transplantation in 45 eyes of children and infants to be $60 \%$. The latter study could not find any statistically significant difference in graft survival between congenital and acquired corneal opacities. Poor prognosis was associated with eyes requiring lensectomy and vitrectomy at the time of penetrating keratoplasty.

Erlich et al reported clear grafts in seven of 16 eyes with Peters' anomaly and in none of eight eyes with congenital glaucoma. ${ }^{9}$ Corneal transplantation for congenital corneal opacities was extended beyond Peters' anomaly to buphthalmic and microphthalmic eyes. ${ }^{11}$ These studies demonstrated that the latter conditions which were considered inoperable can undergo successful corneal grafting. Other authors have reported results of paediatric keratoplasties, but their series were very heterogeneous with respect to preoperative diagnosis and age at the time of surgery. ${ }^{12-17}$

This article describes the outcome of PKP in 58 eyes of 42 patients with congenitally opaque corneas.

\section{Materials and methods}

We reviewed the records of 48 infants and small children who underwent PKP for congenital corneal opacities between January 1983 and December 1992 at the University of California at San Diego. Eyes which had previously undergone PKPs at other institutions were excluded. Fifty eight eyes of 42 patients were included in this study.

\section{PATIENTS}

Preoperative diagnoses included 27 eyes with sclerocornea (total scleralisation of the cornea), 12 eyes with partial sclerocornea (central opacity that extends to part, but not all the limbus), 17 eyes with Peters' anomaly (central corneal opacity), and two eyes with congenital glaucoma.

Fourteen patients had unilateral opacities. Of the 22 patients with bilateral opacities, five were operated only unilaterally because of severe microphthalmia of the fellow eye or life threatening cardiac malformation. Five eyes were operated in monocular patients. Five eyes were markedly buphthalmic, and microphthalmia was associated in 10 eyes with sclerocornea (seven eyes) or partial sclerocornea (three eyes). Surgery was performed between 5 days and 65 months of age. Twenty of the procedures were carried out in the first 2 months of life. The mean age at first surgery was 6.3 (SD 11.9) months. Follow up ranged from 2 to 118 months (37.5 (31)). Two children died in the first 2 years after surgery. All opacities were considered to be of significant size and location 


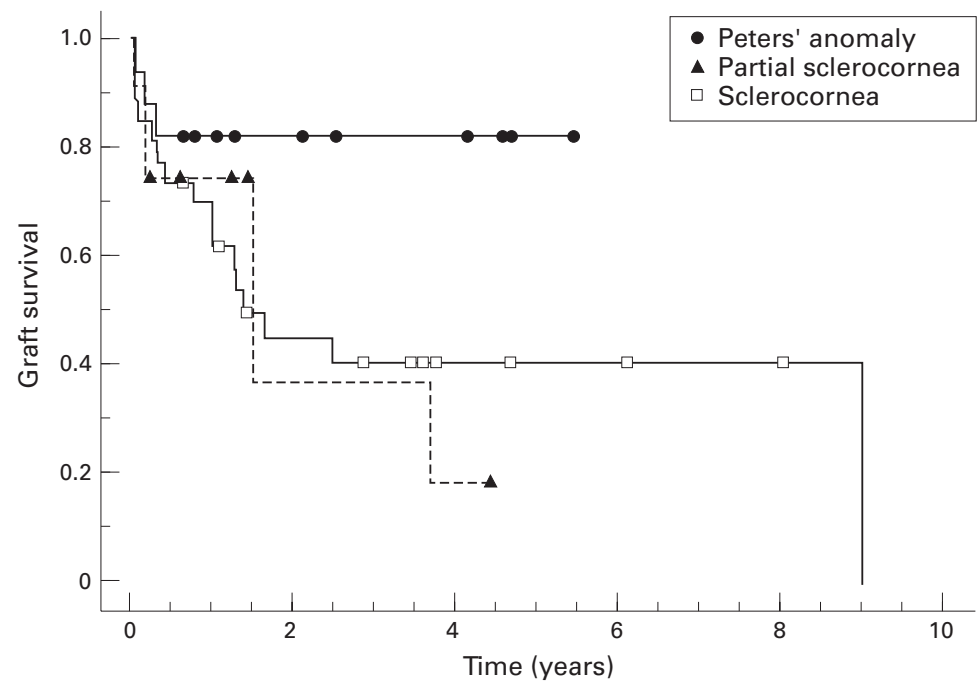

Figure 2 Kaplan-Meier survival curves for the first grafts, presented by different diagnostic groups. Congenital glaucoma (two eyes) have been excluded.

to prevent the development of central vision. The use of intraoperative heparin in those eyes with grossly normal iris and lenses was divided into two groups. One group consisted of 18 eyes in which intraoperative heparin was used at the time of the first penetrating keratoplasty. The second group included the previous 18 eyes which had been operated before the introduction of heparin.

SURGICAL TECHNIQUE

All penetrating keratoplasties were performed by one surgeon (SIB). Thirteen eyes with preoperative glaucoma underwent cyclocryotherapy (nine eyes) or trabeculectomies (four eyes) before PKP. In particularly opaque corneas, retroillumination was performed in the operating room to outline the anatomy of the anterior segment for proper graft centration as well as selection of trephine size. A partial thickness trephination of the recipient bed was performed using a hand held trephine, varying in diameter from 3 to $7 \mathrm{~mm}$. The anterior chamber was entered using a sharp blade. Since 1989 , a solution containing $100 \mathrm{U} / \mathrm{ml}$ of heparin has been injected anterior to the iris immediately after anterior chamber entrance to prevent fibrin formation and subsequent synechiae in all eyes that had a relatively intact iris for both primary and repeat transplantations. The recipient button was then excised with scissors. When present, adhesions to the iris were dissected from the back of the host cornea during its excision rather than directly cutting iris strands which results in bleeding. Lensectomy and anterior vitrectomy were performed when the lens was abnormal in size, location, or transparency.

The donor tissues were from individuals older than 18 years and were always at least 1 $\mathrm{mm}$ larger than the recipient bed. The transplants were sutured in place using interrupted 10-0 nylon sutures after reforming the anterior chamber with balanced salt solution (Alcon, Fort Worth, TX, USA). A single 10-0 Prolene or nylon running suture was used in the first seven grafts. This suturing technique proved unsatisfactory because of frequent uneven healing of the wounds, and was subsequently discontinued. Viscoelastics (Healon, Pharmacia, Uppsala, Sweden) were used in two cases to deepen the chamber. In both instances the presence of the material seemed to prevent tight wound closure.

Approximately one half of the eyes with sclerocornea had oval shaped corneas created by anterior insertion of the conjunctiva onto the superior and inferior scleralised cornea. In these eyes, the operative technique included recessing the conjunctiva $2-3 \mathrm{~mm}$ from the true limbus and removing subconjunctival tissue so that the conjunctiva remained recessed without sutures.

\section{POSTOPERATIVE MANAGEMENT}

Postoperatively, patients received $1 \%$ prednisolone acetate drops at hourly intervals for at least 6 months as well as topical antibiotics until the sutures were removed. Usually the $1 \%$ prednisone was continued hourly for 6 months. Thereafter, topical prednisone was applied every 2 hours beginning at 1 year postoperatively. The interval was increased gradually until 18 months of age when prednisone was stopped. The parents were told to initiate or increase to hourly intervals whenever the child had fever as well as having coincidental ophthalmic examination. Antiglaucoma medications were applied whenever necessary. No systemic immunosuppressive therapy was given.

The eyes were examined almost daily with a flashlight and occasionally under sedation with a microscope or portable slit lamp until suture removal, usually between 1 and 3 weeks postoperatively for sclerocorneas and 6 to 12 weeks for Peters' anomaly. Infants older than 14 months could often be examined at the slit lamp. In all cases, special attention was given to recognise micro abscesses and loose sutures with small mucous strands which could provoke graft rejection.

Loose sutures were removed under either sedation with chloral hydrate or general anaesthesia at the day of diagnosis and micro abscesses were opened with forceps. Repeat penetrating keratoplasty was usually performed within a month after graft failure. In the event of a non-healing epithelial defect in the immediate postoperative period of eyes with sclerocornea, regrafting of the eye was elected since host resurfacing was always followed by vascularisation and surface opacification. When epithelial erosions occurred later, it was usually because of lagophthalmos and in these eyes, the lids were patched closed during sleep or partial tarsorrhaphies were occasionally performed. Glasses were prescribed on the basis of retinoscopy, and patching of the best eye was begun in the first few days after surgery. Parents were instructed to recognise loose sutures or graft rejection with the aid of a flashlight.

Overall, failures were defined by repeated graft rejections, graft failure in patients who could not remain in the area to undergo repeat surgery, or loss of vision after inoperable retinal 


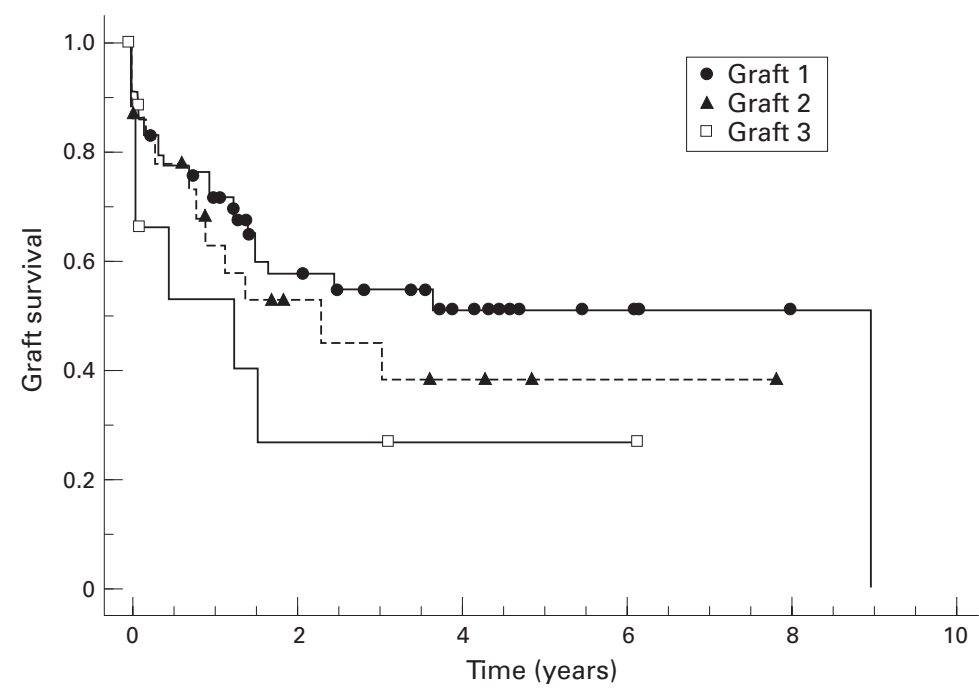

Figure 1. Kaplan-Meier survival curves of graft survival for the first, second, and third penetrating keratoplasty. The symbols represent end of follow up.

detachment. Binocularity was estimated in infants after surgery for unilateral disease by the alignment as well as the fixation response to 4 dioptre prism. In older children a stereopsis test was utilised, as well as the Worth four dot test.

STATISTICAL ANALYSIS

Data were collected until March 1993. Statistical analysis on graft survival was performed using the Kaplan-Meier survival analysis. ${ }^{18}$ The Mantel-Haenszel approach was used to compare survival curves. ${ }^{19}$

\section{Results}

Kaplan-Meier graft survival curves for the first, second, and third penetrating keratoplasties are shown in Figure 1. Primary grafts were clear in $75 \%(\mathrm{SE} 6 \%$ ) at 1 year and $57 \%(7 \%)$ at 2 years, second grafts $67 \%(10 \%)$ and $51 \%$ $(11 \%)$, respectively. Finally, third grafts were clear in $56 \%(16 \%)$ at 1 year. The traces demonstrate that the regrafted eyes had a higher chance of failure. Although the curves show a trend towards better prognosis for the first grafts, but less so for second and third grafts, the differences failed to reach statistical significance.

Overall, $83 \%$ or 48 grafts in the 58 eyes remained transparent throughout the entire follow up period. The latter included primary as well as regrafts. Fifty three eyes had a follow up of between 12 and 118 months. The remaining five eyes were lost to follow up before 7 months postoperatively. Mean follow up was of 40 (SD 29) months. Twenty three eyes were regrafted between 2 weeks and 110 months after the first penetrating keratoplasty. Ten of these 23 eyes had more than two penetrating keratoplasties. The total number of penetrating keratoplasties, both primary and repeat procedures, was 97 . The indications for the first regraft included graft failure (17 eyes), non-healing epithelial defect in the early postoperative course (three eyes), bacterial corneal ulcer (one eye), use of a temporary keratoprosthesis for retinal detachment repair (one eye), and retrocorneal membrane (one eye). Two episodes of graft rejection occurred following suture removal. Several associated systemic and ocular anomalies were noted. All have been reported in previous studies. ${ }^{20-25}$ The most common anomalies were cardiac malformations, mental retardation, dysmorphic traits, and Goltz syndrome. One case of chromosome $6 \mathrm{p}$ deletion was noted. No other chromosomal aberrations were detected in the 10 children who had undergone genetic testing. Family history was positive for sclerocornea $(n=1)$, iris coloboma $(n=1)$, and anophthalmia $(n=1)$. The mean age of the mothers in the series was 30.5 (SD 6) years (range 21-40) and of the fathers 34.3 (7) years (range 22-50).

There were no corneal opacities in the 67 siblings born before or the 23 siblings born after the patients in this series. Three parents, all mothers, had congenital ocular alterations. One had unilateral sclerocornea, one had a unilateral iris coloboma, and the last had bilateral microcorneas.

\section{DIAGNOSTIC GROUPS}

The 17 eyes with Peters' anomaly displayed relatively little clinical pathology. In 11 of the eyes, there were obvious iris adhesions to the opacified central cornea. In the remaining six eyes, there were no apparent central adhesions. In two eyes, the lens appeared to be adherent to the central corneal opaque zone. In both of these eyes, the anterior lens capsule was apparently intact since it was possible to bluntly dissect the lens from the cornea without rupturing the anterior capsule. None of the eyes with Peters' anomaly had preoperative glaucoma, and none needed lensectomy and anterior vitrectomy during the first keratoplasty. Two eyes developed glaucoma towards the end of the first postoperative year. The 12 eyes with partial sclerocornea had a similar incidence of central anterior synechiae, but had relatively more severe anterior segment pathology - that is, one eye had an iris coloboma and two had preoperative glaucoma.

In total sclerocornea, the clinical pathology was more extensive. Thirteen of the 27 sclerocorneas appeared to be oval, with the longest meridian in the horizontal axis. This oval appearance was apparently due to a more anterior insertion of the conjunctiva inferiorly and superiorly. Although 12 eyes had apparently normal irides and lenses, the remaining 15 eyes had anomalies which varied from partially developed lenses and/or irides to complete absence of the lens and the presence of only a film of iris pigment. In two eyes, lens remnants were found within the corneal stroma, and no evidence of lens material was observed clinically or histologically in two eyes. Ten of the sclerocornea eyes had preoperative glaucoma. Twelve eyes underwent lensectomy and anterior vitrectomy during the first keratoplasty.

Analysed according to diagnoses, Peters' anomaly had the best prognosis with $100 \%$ success, followed by partial sclerocornea at $83 \%$, and sclerocornea at $70 \%$. Figure 2 shows the Kaplan-Meier curves for these diagnoses. Differences in the graft survival between 


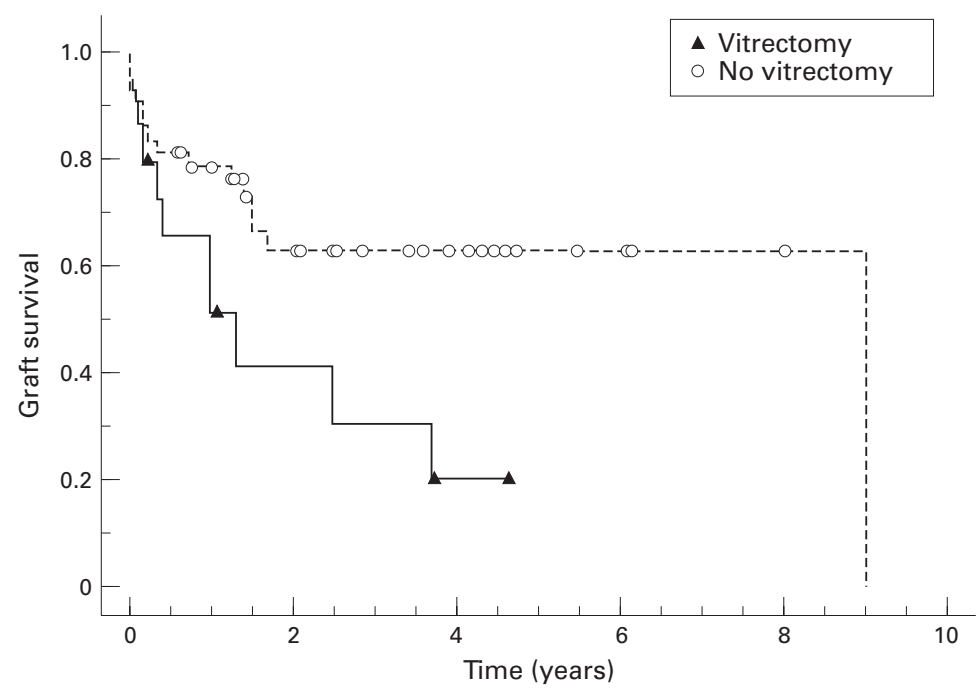

Figure 3 Kaplan-Meier survival curves of grafts with and without lensectomy and anterior vitrectomy at first surgery. Mantel-Haenszel approach showed a significant difference in graft survival between the two groups $(p=0.018)$.

Peters' anomaly and sclerocornea were significant $(p=0.038)$. The two eyes with bilateral congenital glaucoma had transparent grafts 52 and 47 months after the primary keratoplasty.

VITRECTOMY AT FIRST SURGERY

Fifteen eyes (12 in sclerocornea, three in partial sclerocornea) underwent anterior vitrectomy with or without lensectomy at the first keratoplasty. Figure 3 shows the survival curves for eyes with and without lensectomy at first keratoplasty. The difference in graft survival is statistically significant $(p=0.018)$. Eyes with primary lensectomy and anterior vitrectomy had a $65 \%(12 \%)$ chance of a clear graft at 1 year, compared with $78 \%$ (6\%) for keratoplasties without additional procedures. The incidence of vitrectomy appeared related to the anatomical alterations - that is, in all of the eyes the iris was absent and the lens was either underdeveloped or absent. Twelve of these eyes had either preoperative or postoperative glaucoma.

FAILURES, COMPLICATIONS

Ten eyes failed after both primary and repeat keratoplasties. Reasons for irreparable failure were repeated graft rejections in four eyes, inoperable retina detachment in three eyes, loss of transparency due to chronic surface problems related to lagophthalmos in one eye, and the necessity to leave the vicinity of the surgeon in one eye and a bacterial ulcer with perforation in one eye. Fourteen eyes developed postoperative glaucoma from anterior synechiae and 11 of those were treated with cyclocryotherapy or other surgical procedures to control the intraocular pressure. Following cryotherapy, seven eyes in this subgroup developed cataracts. Five eyes developed band keratopathy after cyclocryotherapy for preoperative or postoperative glaucoma. Corneal ulcers and failed grafts occurred in two eyes after trabeculectomy combined with the operative and postoperative administration of 5-fluorouracil. Cataract developed in 12 eyes,
11 of which underwent extracapsular cataract extraction (without intraocular lens implantation) between 1 month and 4.5 years after the initial surgery.

When donor epithelium was absent on the first postoperative day, epithelialisation was slow and incomplete in three eyes with sclerocornea. This was presumed to be due to poor resurfacing by conjunctival epithelium that covers the sclerocornea eye. These eyes required regrafts. Epithelial erosions leading to regrafts occurred in two additional eyes with lagophthalmos which was the result of glaucomatous enlargement of the eyes. The regrafts were facilitated by partial tarsorrhaphies.

\section{HEPARIN}

Beginning in 1989, heparin was utilised intraoperatively in order to prevent fibrin formation and the subsequent development of anterior synechiae and postoperative glaucoma. We compared 18 eyes in which heparin had been used with 18 earlier primary grafts without heparin. Heparin use did not cause intraoperative haemorrhages or any other complications or side effects. Although four heparin treated eyes had one observable anterior synechiae of less than one clock hour in width, none developed postoperative glaucoma. In the untreated group, seven of 18 eyes developed postoperative glaucoma. These appeared to result from broad anterior synechiae which, over time, spread to seal a majority of the anterior chamber. None of the heparin treated eyes had graft failure. Four of the 18 eyes without heparin had graft failures.

\section{VISUAL ACUITY}

Visual acuity was difficult to assess owing to the very young age of most of the patients, the relative high percentage of children with marked mental retardation (14 eyes, 10 out of 42 children), and the fact that 18 came from out of state. The best achieved vision at last visit was $20 / 50$ in three eyes. Eight eyes had visual acuities of 20/400. Seven eyes had quantifiable visual acuities ranging from $20 / 60$ to $20 / 200$. All recorded refractions were myopic and ranged from -2.25 to -22 dioptres. Three were blind as a result of retinal detachment. The rest of the eyes had sufficient vision to recognise various sized animals in reading books and to recognise figures when they left San Diego, but their present visual acuities have not been communicated to the authors. Three of the patients with unilateral disease achieved some degree of binocularity. Two showed fusion movements when a 4 dioptre prism was placed over either eye. The third patient's corrected refractive error was -22 in the operated eye and +6 in the unoperated eye. In spite of this anisometropia, the patient could correctly identify four dots at near and demonstrated stereopsis with the $3 \mathrm{D}$ fly. The latter patient's surgery was at 12 days while the other two underwent their surgery at 2 and 8 weeks of age. 


\section{Discussion}

This study shows that the overall success rate of penetrating keratoplasty for congenital corneal opacities in our patient population was $83 \%$. The overall success rate by anatomical diagnosis was best for Peters' anomaly with $100 \%$ of clear grafts at last visit, followed by partial sclerocornea ( $83 \%)$, and by sclerocornea $(70 \%)$. It further indicates that the chance of a clear first graft at 1 year was best in Peters' anomaly (82\%), followed by partial sclerocornea $(73 \%)$ and sclerocornea $(70 \%)$. Most graft failures or regrafts occurred in the first year. The better prognosis in Peters' anomaly and partial sclerocornea relates to the lack of severe intraocular anomalies and consequent lower incidence of early glaucoma as well as the need for lensectomy and anterior vitrectomy at first surgery. The poorer prognosis for sclerocornea is probably related to the higher incidence of major anatomical alterations in this condition. Angle anomalies resulting in primary glaucoma and, often, buphthalmos occurred in almost half of the eyes with sclerocornea. Surgery was complicated in eyes with primary glaucoma because these usually had thinner corneas from stretching of the eye. In addition, 12 out of 27 of the eyes with sclerocornea needed anterior vitrectomy with or without lensectomy at first surgery because of absence or alteration of the lens, which complicated the surgical procedure. Vitrectomised eyes had a significant higher rate of repeat keratoplasties. A significant reason for the failed grafts were the high incidence of glaucoma and the complications from treatment. Further, it was only in eyes that had vitrectomies that retrocorneal connective tissue developed which led to graft failure in four eyes. Finally, sclerocorneas appeared to heal approximately twice as fast as the nonvascularised corneas. The surgical procedure in all eyes was complicated by a phenomenon almost unique to infants - that is, massive outpouring of fibrin upon entering the anterior chamber, promoting broad anterior synechiae, resulting in postoperative glaucoma in many of the earlier cases in the series. The management of postoperative glaucoma was unsatisfactory since medical treatment frequently failed. When surgery was required to lower the intraocular pressure, cyclocryotherapy was the preferred procedure in this patient group. This preference was based on our previous poor results of filtering procedures in children's eyes with congenital corneal opacities and on the development of severe bacterial ulcers in two eyes which had undergone trabeculectomies with 5-fluorouracil. More recently, we have had fairly impressive success with filtering procedures, and if the success is lasting will be the subject of a future study. Although the preplacement of a Molteno tube at time of the first keratoplasty has been advocated, we have no experience with this procedure. ${ }^{26}{ }^{27}$ However, because many of the eyes in this series had either sclerocornea with severe anterior segment abnormalities or microphthalmos, it would appear that tubes for these eyes would present a significant challenge.
The use of a $100 \mathrm{U} / \mathrm{ml}$ heparin solution to irrigate the anterior chamber to prevent fibrin formation and subsequent anterior synechiae appeared to uniformly prevent postoperative glaucoma. Heparin has been used for decades in our and other laboratories to decrease fibrin production in corneal transplantation of rabbit eyes. It also has been used as an adjunct to vitrectomy in diabetic eyes for the same reasons. ${ }^{28-30}$ The use of heparin in eyes in which iris tissue was present improved the clinical outcome, as shown in the comparison between the eyes treated with heparin with those which were not. The follow up for the heparin group was shorter, but was long enough to assess the role of heparin in preventing broad synechiae, since synechiae formation occurs in the early postoperative period and can therefore be detected in the first months after surgery. The heparin treated eyes had a clinically significant lower rate of postoperative complicationsthat is, less anterior synechiae, postoperative glaucoma, and repeat keratoplasty. The use of heparin obviated the need for viscoelastics and seemed to shorten the procedure.

Sutures in a vascularised infant cornea incite an inflammatory reaction which will lead to graft rejection unless the sutures are removed in the first postoperative weeks, as previously reported. ${ }^{1}$ This inflammatory response to sutures was more frequent in eyes with the oval shaped corneas of sclerocorneas where the suture tracks approximated or ran through the conjunctiva. Recessing the superior and inferior conjunctiva resulted in more even healing and allowed a delay in suture removal until 2-4 weeks postoperatively. Despite the anatomical and surgical problems associated with sclerocornea, there was still an overall success rate of $70 \%$. This success rate was even more significant since nine of the 27 eyes also had buphthalmos, congenital staphylomas, or marked microphthalmia.

Our results of surgery (for unilateral corneal opacities) indicate a strong justification for carrying out surgery at any time in bilateral cases and during the first 3 months with unilateral involvement. All but one of the eyes with unilateral opacities achieved, postoperatively, vision that allowed recognition of figures in children's books and to play unaided in unfamiliar surroundings. In two very cooperative children where surgery was carried out between 12 days and 9 weeks after birth, we were able to suggest some degree of central fusion in two eyes and peripheral fusion in one eye. The visual acuity in all three eyes was at least 20/60. Recently, Cameron has published a case of unilateral Peters' anomaly with 20/40 vision and peripheral fusion 49 months after penetrating keratoplasty. ${ }^{31}$ These results, in addition to the recent literature relating to the results of early unilateral congenital cataract surgery, ${ }^{32}$ indicate corneal transplantation in the first weeks of life with unilateral disease. However, even surgery in the first 3-4 months will provide the infant with a spare eye with visual function that usually allows independent ambulation. Further, as Stulting et al noted previously, infants that had transparent grafts 
for even a few months maintained useful vision after the grafts failed. ${ }^{2}$ In a multicentre study, Dana et al also found that multiple surgical procedures, like vitrectomies or regrafts, contributed to worse graft survival in paediatric keratoplasties. $^{3}$ In their heterogenic group 81 keratoplasties were performed for congenital opacities and showed a graft survival of $80 \%$ at 1 year and approximately $65 \%$ at 2 years. These results are very encouraging but are difficult to compare with our report, because of the smaller proportion of sclerocorneas in their series.

In no instance did any of the siblings born before or after the patients in this series develop congenital corneal opacities. The latter suggests that rather than an autosomal recessive inheritance pattern, congenital corneal opacities seem to occur sporadically. The parental age may be significant. Thirty three per cent of the mothers were older than 32 years of age and it is well known that more congenital anomalies occur as a result. Further, $46 \%$ of the fathers were older than 35 . The latter could indicate that the corneal opacities represented a new mutation and that the infants may have acquired a dominant trait. This possibility was further suggested by the fact that the mother of one of the infants with unilateral total sclerocornea also had unilateral complete sclerocornea. To the best of our knowledge, the results of this series of transplantation of congenitally opaque corneas are the best published. This is also the largest series of PKPs for congenital opacities performed by a single surgeon. Further, it contains the largest series of eyes with sclerocornea, as well as the most diverse spectrum of anomalies. We believe the success rate reported here is the result of the use of intraoperative heparin, improved surgical technique for sclerocornea, the avoidance of suture related complications with early suture removal, vigorous treatment with topical corticosteroids, and total parental commitment. The postoperative course can be quite complex, resulting in a high percentage of regrafts, especially in eyes with more anterior segment alterations.

We conclude that penetrating keratoplasty for congenital corneal opacities has the potential for excellent long term results and should be performed in the first weeks of life in both bilateral and unilateral cases.

Supported by Research to Prevent Blindness.

1 Brown SI, Salamon SM. Wound healing of grafts in congenitally opaque corneas. Am $\mathcal{f}$ Ophthalmol 1983;95:641-4
2 Stulting RD, Sumers KD, Cavanagh HD, Waring GOI, Gammon JA. Penetrating keratoplasty in children. OphthalGammon JA. Penetrating

3 Dana M-R, Moyes A, Gomes J, Rosheim K, Schaumberg D, Laibson $\mathrm{P}$, et al. The indications for and outcome in pediatric keratoplasty: a multicenter study. Ophthalmology 1995;102:1129-38.

4 Brown SI. Corneal transplant in the anterior chamber cleavage syndrome. Am f Ophthalmol 1970;70:942-4.

5 Wood TO, Kaufman HE. Penetrating keratoplasty in an infant with sclerocornea. Am f Ophthalmol 1970;70:60913.

6 Brown SI. Corneal transplantation of the infant cornea. Trans Am Acad Ophthalmol Otol 1974;78:461-6.

7 Waring GOI, Laibson PR. Keratoplasty in infants and chilWaring GOI, Laibson PR. Keratoplasty in infants and chil
dren. Trans Am Acad Ophthalmol Otol 1977;83:283-96.

8 Schanzlin DJ, Goldberg DB, Brown SI. Transplantation of congenitally opaque corneas. Ophthalmology 1980;87: congenitally

9 Erlich CM, Rootman DS, Morin JD. Corneal transplantation in infants, children and young adults: experience of the Toronto Hospital for Sick Children. Can $\mathcal{F}$ Ophthalmol 1991;26:206-10.

10 Frucht-Pery J, Feldman ST, Brown SI. Transplantation of congenitally opaque corneas from eyes with exaggerated buphthalmos. Am f Ophthalmol 1989;107:655-8.

11 Feldman ST, Frucht-Pery J, Brown SI. Corneal transplantation in microphthalmic eyes. Am f Ophthalmol 1987;104: 164-7.

12 Legeais JM, Jobin D, Pouliquen Y. Kératoplasties chez l'enfant. Analyse sur 10 ans de 127 yeux opérés. $\mathcal{F ~ F r}$ Ophtalmol 1990;13:116-20.

13 Cowden JW. Penetrating keratoplasty in infants and children. Ophthalmology 1990;97:324-9.

14 Beauchamp GR. Pediatric keratoplasty: problems in management. F Pediatr Ophthalmol Strabismus 1979;16:388-94.

15 Gordon YJ, Mokete M. Penetrating keratoplasty in children. 7 Pediatr Ophthalmol Strabismus 1979;16:297-300.

16 Völker-Dieben H. Keratoplasty in children. Doc Ophthalmol 1984;57:79-91.

17 Schönherr U, Kuchle M, Lang G, Naumann G. Keratoplastik im Kindesalter. Bericht über 71 Keratoplastiken. Klin Monatsbl Augenheilkd 1993;203:167-73.

18 Kaplan EL, Meier P. Nonparametric estimation from incomplete observations. f Am Stat Assoc 1958;53:457-81.

19 Mantel N, Haenszel W. Statistical aspects of the analysis of data from retrospective studies of disease. 7 Natl Cancer Inst 1959;22:719.

20 Heon E, Barsoum-Homsy M, Cevrette L, Jacob JL, Milot J, Polemeno R, et al. Peters' anomaly. The spectrum of associated ocular and systemic malformations. Ophthalmic Paediatr Genet 1992;13:137-43.

21 Mayer U. Peters' anomaly and combination with other malformations. Ophthalmic Paediatr Genet 1992;13:131-5.

2 Moriarty A, Kerr-Muir M. Sclerocornea and interstitial deletion of the short arm of chromosome 6-(46XY del[6][p22p24]). F Pediatr Ophthalmol Strabismus 1992;29: $177-9$.

23 Goldstein J, Cogan D. Sclerocornea and associated congenital anomalies. Arch Ophthalmol 1962;67:761-8.

24 Kivlin J, Fineman R, Crandall A, Olson R. Peters' anomaly as a consequence of genetic and non-genetic syndromes. Arch Ophthalmol 1986;104:61-4.

25 Van Schooneveld M, Dellerman J, Beemer F, BleeckerWagenmakers E. Peters'-plus: a new syndrome. Ophthalmic Paediatr Genet 1984;4:141-6.

26 Parmley V, Stonecipher K, Rowsey J. Peters' anomaly: a review of 26 penetrating keratoplasties in infants. Ophthalmic Surgery 1993;24:31-5.

27 Astle W, Lin D, Douglas G. Bilateral penetrating keratoplasty and placement of a Molteno implant in a newborn with Peters' anomaly. Can f Ophthalmol 1993;28:276-82.

28 Johnson RN, Balyeat E, Stern WH. Heparin prophylaxis for intraocular fibrin. Ophthalmology 1987;94:597-601.

29 Johnson RN, Blankenship G. A prospective, randomized clinical trial of heparin therapy for postoperative intraocular fibrin. Ophthalmology 1988;95:312-7.

30 Iverson DA, Katsura H, Hartzer MK, Blumenkranz MS. Inhibition of intraocular fibrin formation following infusion of low-molecular-weight heparin during vitrectomy. Arch Ophthalmol 1991;109:405-9.

31 Cameron J. Good visual results following early penetrating keratoplasty for Peters' anomaly. F Pediatr Ophthalmol Strabismus 1993;30:109-12.

32 Gregg F, Parks M. Stereopsis after congenital monocular cataract extraction. Am f Ophthalmol 1992;114:314-7. 